Psychotherapeut 2022 $\cdot 67: 1-3$

https://doi.org/10.1007/s00278-021-00564-9 Angenommen: 29. November 2021

(๑) The Author(s), under exclusive licence to Springer Medizin Verlag GmbH, ein Teil von Springer Nature 2022
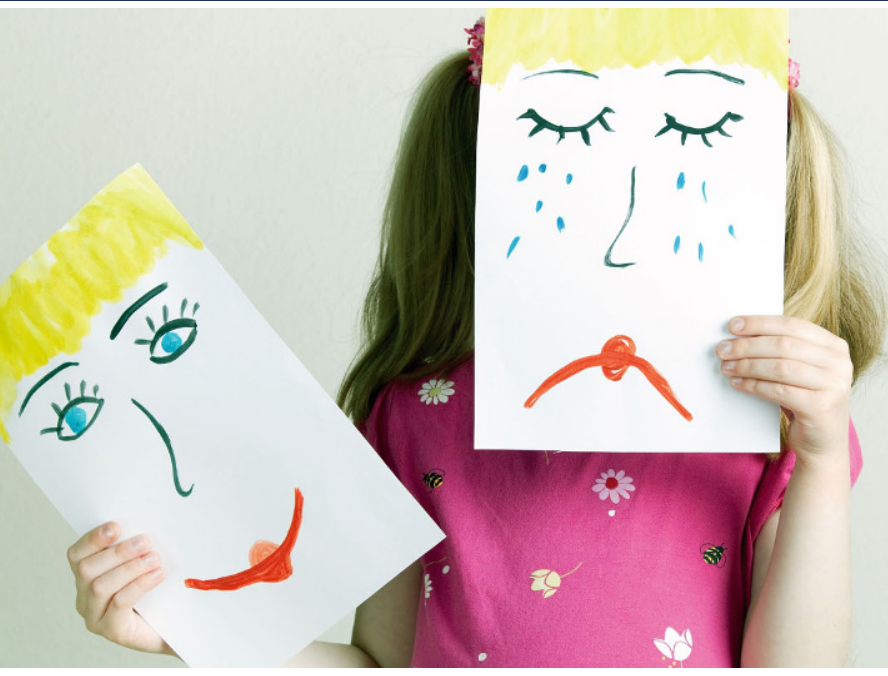

\section{Kinder psychisch kranker Eltern}

\section{Svenja Taubner · Anna Georg}

Institut für Psychosoziale Prävention, Universitätsklinikum Heidelberg, Ruprecht-Karls-Universität Heidelberg, Heidelberg, Deutschland

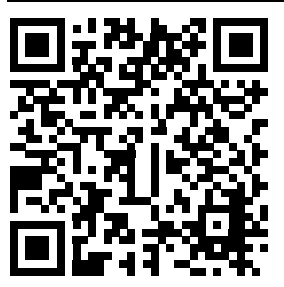

QR-Code scannen \& Beitrag online lesen
Psychisch kranke Eltern und die betroffenen Kinder sind im Fokus dieses Themenheftes, und wir sind sehr froh, Ihnen hier eine große Bandbreite der aktuellen Forschung und Interventionen zu diesem Feld in Deutschland vorstellen zu können. Das Themenheft gliedert sich in Übersichts- und Originalarbeiten, die zunächst die Bedeutsamkeit des Themas unterstreichen und dann Überlegungen anstellen, wie psychisch kranke Eltern und ihre Kinder adäquat unterstützt werden können. Darin nimmt die Psychotherapie eine herausragende Rolle ein, was sowohl für die institutionelle als auch die Psychotherapie in den niedergelassenen Praxen gilt. Das Themenheft gibt Aufschluss über die drängenden Fragen, wie psychische Erkrankungen einen Risikofaktor für die Entwicklung von Kindern darstellen, ob das aktuelle Angebot in Deutschland psychisch kranke Eltern und Kinder erreicht, und ob weitere relevante Angehörige (z. B. das nicht psychisch kranke Elternteil) ebenfalls einbezogen werden können. Die Angebote reichen von allgemeinen Beratungen bis hin zu differenzierten Settings und störungsspezifischen Behandlungen.

Insgesamt findet aktuell der Paradigmenwechsel in den psychosozialen Settings statt, Patient*innen stärker in dem Kontext ihrer Lebenswelt zu betrachten und die Auswirkungen psychischer Erkrankungen auf andere zum Thema der Interventionen zu machen. Damit werden "hidden patients" und psychosoziale Folgen sichtbar gemacht. Wenn dies in unterstützender und nichtstigmatisierender Weise geschieht, kann ein profunder Beitrag zur Prävention von dysfunktionalen familiären Interaktionen und der transgenerationalen Weitergabe psychischer Erkrankungen geleistet werden. Keinesfalls sollen alle psychisch kranken Eltern und ihre Angehörigen unter einen Generalverdacht gestellt werden, sondern differenziert beraten und unterstützt werden.

Sowohl aus Sicht von psychisch erkrankten Müttern als auch aus der Perspektive von Kinderärzt*innen, die im Rahmen von Früherkennungsuntersuchungen auch mit psychisch erkrankten Eltern Kontakt haben, zeigen sich eine erhöhte elterliche 
Belastung und Einschränkungen in den Bereichen Elternkompetenzen, Wahrnehmung der kindlichen Bedürfnisse und Impulsivität in der Eltern-Kind-Beziehung. Alle diese Bereiche können sich potenziell nachteilig auf die kindliche Entwicklung auswirken. Diese Problembereiche zeigten sich in einer Auswertung von Interviews und Fragebogen im Rahmen einer Studie des Nationalen Zentrums Frühe Hilfen, die von van Staa et al. vorgestellt wird. Allerdings werden psychotherapeutische Hilfsangebote nur von einem Drittel der betroffenen Eltern wahrgenommen, wie sich in einer repräsentativen Studie des DJ zeigte, die von Reim et al. in das vorliegende Themenheft eingebracht wurde. Es zeigte sich überdies, dass die Inanspruchnahme therapeutischer Leistungen unabhängig von der von den Eltern wahrgenommenen kindlichen Belastung war, weshalb die Autor*innen von einer unzureichenden Versorgung sowohl der psychisch kranken Eltern als auch von deren Kindern ausgehen.

Neben den psychischen Erkrankungen, die bereits vor der Familiengründung oder Geburt von Kindern bestehen, können Ereignisse und die psychische Verarbeitung der Schwangerschaft, die Entstehung psychischer Störungen während bzw. in der Folge einer Schwangerschaft bewirken. Stuhrmann et al. beleuchten in ihrer Studie, wie sich pränatale schwangerschaftsspezifische Ängste auf das Vertrauen in die eigenen Fähigkeiten als Mutter in der frühen Elternschaft auswirken. Dabei zeigt sich im Rahmen ihrer prospektiven Längsschnittstudie, dass der Effekt von schwangerschaftsspezifischen Ängsten auf eine geringe mütterliche Selbstwirksamkeit durch das Ausmaß der postpartalen Depressivität vermittelt wird. Daher ergibt sich die Bedeutsamkeit der Weiterentwicklung von Behandlungsansätzen mit Beginn in der Schwangerschaft, die sowohl Ängste, Depressivität und mütterliche Selbstwirksamkeit im Kontext einer engen interprofessionellen Zusammenarbeit zwischen eltern- und kindbezogenen Disziplinen adressieren. In einer auf diesen Beitrag folgenden Übersichtsarbeit betrachtet Bindt die psychosozialen Risiken, die mit einer Frühgeburt einhergehen können. Aktuell ist jedes 12. Kind in Deutschland eine Frühgeburt, wobei die Zahlen weiter- ansteigen. Ein höheres psychosoziales Risiko innerhalb dieser Gruppe wird Kindern mit sehr niedrigem Geburtsgewicht zugeschrieben. Für diese Hochrisikogruppe der Frühgeboren wird derzeit ein „Frühgeborenenverhaltensphänotyp" diskutiert, der mit Symptomen von Angst-, Aufmerksamkeits- und Autismus-Spektrum-Störungen einhergehen kann. In dem Beitrag werden die besonderen peri- und postpartalen Risiken für Eltern und Kind und deren Interaktion nachvollziehbar beschrieben. Das sog. Känguru-Pflegemodel könnte eine Alternative darstellen, die besondere Risiken der intensivmedizinischen Betreuung abmildern könnte, wenn dies medizinisch überhaupt indiziert ist.

Im Rahmen des familienzentrierten Ansatzes zu Beratung und Therapie von Familien mit psychisch kranken Eltern finanziert der Innovationsfonds des Gemeinsamen Bundesausschusses der Krankenkassen aktuell das Verbundprojekt CHIMPSNET. Wiegand-Greve erläutert den Ansatz des bundesweiten Verbunds aus einer Forschungs-, Versorgungs- und Implementierungsperspektive. Zentral ist bei CHIMPS-NET, den befristeten Projektcharakter vieler Unterstützungsangebote für psychisch kranke Eltern zu überwinden und eine familienorientierte Regelversorgung für die Kinder und Jugendlichen psychisch erkrankter Eltern in Deutschland zu etablieren. Im Rahmen des Verbundprojektes sind auch die von Holl et al. beschriebene Entwicklung und erste Pilotierung zweier Screenings für minderjährige Kinder psychisch erkrankter Eltern angesiedelt. Die Screeninginstrumente sind spezifisch für den Elternbericht in der Erwachsenenpsychiatrie konzipiert. Psychiatrische Behandler erhalten eine erste Einschätzung zum Unterstützungsbedarf der Familie und der Kinder mithilfe eines Ampelsystems. So können weitere Schritte zur integrierten Versorgung der Familie eingeleitet und negativen Folgen für diese Kinder vorgebeugt werden.

Ein Ansatzpunkt zur Stärkung einer familienorientierteren Versorgung in der Psychiatrie ist die Schulung von medizinischen Fachkräften. Taubner et al. beschreiben die Entwicklung und die Evaluation eines Trainings für medizinische Fachkräfte. Das Projekt soll die Implementierungsprozesse der CHIMPS-
NET-Interventionen stärken. Das Training zielt darauf ab, eine bifokale Perspektive fördern, d.h., sowohl die psychisch kranken Eltern als auch die Kinder und weitere Familienmitglieder in den professionellen Blick zu nehmen und ein klinikenübergreifendes und abgestimmtes Behandlungsangebot zu etablieren. In den teilnehmenden Klinken zeigte sich einerseits eine bereits etablierte Familienorientierung bei einigen Fachkräften. Die qualitative Auswertung der Implementationsprotokolle wies allerdings auf einen hohen Bedarf nach institutionsübergreifender Vernetzung und klaren Standardprozeduren, z. B. im Umgang mit Kindeswohlgefährdung, hin.

Obschon bei allen Kindern von Eltern mit psychischen Erkrankungen ein erhöhtes Risiko vorliegt, stellen Kinder von Eltern mit einer Borderline-Persönlichkeitsstörung (BPS) eine besonders belastete Risikogruppe dar. Seehausen et al. stellen die Intervention "Borderline und Mutter sein" vor, die sich der Förderung der Erziehungskompetenz von Müttern einer BPS widmet. Unter Berücksichtigung störungsspezifischer Schwierigkeiten in der MutterKind-Interaktion sollen hier dysfunktionale Erziehungsstrategien durch positive Erlebens- und Verhaltensmuster ersetzt werden.

Zwei Beiträge thematisieren den Einbezug weiterer Angehöriger in die Therapie von beispielsweise psychisch erkrankten Eltern oder ihren Kindern. Müller und Taubner beschreiben das Projekt "Mit Papa geht es besser", in dem die Wirksamkeit des Einbezugs der Väter in die stationäre Mutter-Kind-Therapie bei postpartalen psychischen Störungen untersucht wird. Die Ergebnisse der hier veröffentlichten Pilotstudie des Projektes weisen auf tendenzielle Verbesserungen in den Zielkriterien der Intervention hin. Die Verbesserungen zeigten sich insbesondere in dem Bereich der Partnerschaftszufriedenheit. Zuletzt thematisiert der Beitrag von AguilarRaab, Georg und Kolleg*innen das interdisziplinäre Versorgungsangebot am Zentrum für Psychosoziale Medizin des Universitätsklinikums Heidelberg. Das Konzept der 2020 gegründeten interdisziplinären Arbeitsgruppe "Familiengesundheit" wird vorgestellt und - bezogen auf die 4 Fokusse "Kinder und Jugendliche ", "Elternschaft", 
„Familien“ und „Paare“ - die Umsetzung des psychosozialen Versorgungskonzept dargestellt. Mit den vielseitigen Angeboten soll der sozialen Eingebundenheit von Patient*innen gerecht werden und eine effiziente Behandlung und Genesung ermöglicht werden.

Die vielseitigen Arbeiten in diesem Themenheft zeugen von einer neuen Bewegung in der deutschen Versorgungslandschaft, hin zu einer familienorientierteren und präventiv auf das kindliche Wohl ausgerichteten psychotherapeutischen Praxis, die wir sehr begrüßen. Kinder psychisch kranker Eltern und weitere Angehörige besser zu versorgen und eine erhöhte Sensibilität für die Eltern-Kind-Beziehung im Versorgungssystem zu etablieren, könnte weitreichende positive Auswirkungen auf die nachfolgende Generation mit sich bringen. Es bleibt, die Ergebnisse einiger dieser Studien und Vorhaben und vieler anderer Bemühungen, die in diesem Heft keinen Platz fanden, abzuwarten, um zu bemessen, wie gut wir diesen Zielen näherkommen und inwieweit sich die Versorgungsrealität nachhaltig verändern lässt.

\section{Korrespondenzadresse}

\section{Prof. Dr. phil. Svenja Taubner}

Institut für Psychosoziale Prävention, Universitätsklinikum Heidelberg, RuprechtKarls-Universität Heidelberg

Bergheimerstr. 54, 69115 Heidelberg, Deutschland

Svenja.Taubner@med.uni-heidelberg.de

Interessenkonflikt. S. Taubner und A. Georg geben an, dass kein Interessenkonflikt besteht.

\section{Ich weiß, was du sagen wirst!}

Wissen Sie manchmal auch ganz genau, was Ihr Gegenüber gleich sagen wird? Tatsächlich denkt unser Gehirn immer schon einen Schritt voraus. Aber wieso?

Forschende der Universität zu Lübeck und des Max-Planck-Instituts für Kognitionsund Neurowissenschaften haben nun entschlüsselt, wie es das Gehirn schafft, die richtigen Informationen für das »Voraushören« auszuwählen.

In einem Experiment, das den Herausforderungen des alltäglichen Sprachverstehens nachempfunden war, hörten Proband*innen der Literaturnobelpreisträgerin Herta Müller rund eine Stunde aufmerksam dabei zu, wie sie im Plauderton von ihrer Kindheit im rumänischen Banat erzählte. Ein Magnetresonanztomograph zeichnete dabei fortlaufend die Aktivität im gesamten Gehirn der Zuhörenden auf. Menschen speichern ein soeben gehörtes Wort in der Hörrinde im Schläfenlappen und einen langen Satz im höher gelegenen, nachgeschalteten Scheitellappen.

Auch artifizielle Netzwerke aus dem Bereich des Maschinellen Lernens nutzen diese Grundprinzipien des menschlichen Gehirns und speichern das Gesagte in aufeinander aufbauenden, zunehmend abstrakten Verarbeitungsstufen. Mittlerweile sind sie in der Vorhersage von Sprache genauso gut wie Menschen. Auch gut trainierte artifizielle Netzwerke bekamen die Aufgabe, Herta Müllers Erzählung zu- und vorauszuhören. Den Kniff ihrer Studie beschreibt Studienleiterin Lea-Maria Schmitt so: „Indem wir vergleichen, wie Maschine und Mensch einen bestimmten Textabschnitt "bearbeiten", können wir von den bekannten und leicht zu überprüfenden Berechnungen in artifiziellen Netzwerken auf noch unbekannte Verarbeitungsstrategien im Gehirn schließen."

In der Auswertung machten die Forschenden zunächst die Entdeckung, dass Hirnregionen fernab der Hörrinde im Scheitellappen bei jenen Textstellen eine stärkere Aktivierung zeigten, bei denen sich auch eine höhere Verarbeitungsstufe des artifiziellen Netzwerks überrascht von Herta Müllers nächster Äußerung zeigte. Dies verdeutlicht, dass in verschiedenen Hirnarealen unterschiedlich lange Gesprächsinhalte genutzt werden, um eine Vorhersage zu machen und diese mit anderen Hirnarealen abzustimmen. Erst eine solche Segmentierung von Gesprächen erlaubt es den Zuhörenden, einige Gesprächsabschnitte für die Vorhersage heranzuziehen, aber andere (bestenfalls unwichtige) zu ignorieren. Die "Vorhersagehierarchie« in Schläfen- und Scheitellappen konnten die Forscher*innen allerdings nur dann finden, wenn das artifizielle Netzwerk die Gesprächsinhalte in einzelne Ereignisse unterteilte. So werden im Schläfenlappen kurze, gerade erst beschriebene Ereignisse für die Vorhersage genutzt - etwa wenn Herta Müller in den blau-schwarzen Weintrauben (besser gesagt Tintentrauben) im Garten ihrer Eltern die Farbe der Nacht erkennt.

Im Scheitellappen werden hingegen längere, weiter zurückliegende Ereignisse hinterlegt - etwa wenn Herta Müller auf dem Weg zum Elternhaus einen ausgiebigen Spaziergang durch die Straßen ihres Heimatdorfs macht. Diese »ereignisbasierte« Vorhersagehierarchie im Gehirn kann nicht nur sehr viele verschiedene Gesprächsinhalte vorhalten, sondern muss diese auch seltener, nämlich nur am Ende eines Ereignisses, aktualisieren. „Es könnte genau solch eine reichhaltige und doch recheneffiziente Netzwerkarchitektur sein, mit der unser Gehirn beim Zuhören genaue und schnelle Vorhersagen treffen kann", erklärt Forschungsgruppenleiter Jonas Obleser. Im Alltag unterstützt uns diese Hirnfunktion vor allem bei der erfolgreichen Kommunikation mit anderen und könnte künftig auch helfen, die Kommunikation zwischen Menschen und Maschinen wie Siri oder Alexa weiter zu verbessern.

Originalpublikation: L.-M. Schmitt, J. Erb, S. Tune, A. U. Rysop, G. Hartwigsen, J. Obleser, Predicting speech from a cortical hierarchy of event-based timescales. Science Advances 7, eabi6070 (2021). (doi/10.1126/sciadv.abi6070)

Quelle: Universität Lübeck (www.uniluebeck.de [07.12.2021]) 